\title{
Notas territoriales y estadística en el software " $R$ ": entender los distritos electorales en México
}

\author{
Luis Alberto Luna Gómez ${ }^{1}$
}

\begin{abstract}
Resumen
México se divide en 32 estados que componen la Federación. En los 32 estados están localizados los 300 distritos que constituyen parte del poder Legislativo. En los años 1996 y 2005 se reconfiguró su forma lo que impactó en su estructura. Interesa mostrar que la redistritación no es un asunto de forma sin contenido, puesto que una modificación en la forma del espacio es una modificación en la esencia, es decir, es una acción técnica que impacta en la estructura de la geografía de acciones sociales y el paisaje de los recursos, por lo tanto, es una acción improductiva que impacta valorizando o desvalorizando el espacio de las acciones productivas. En caso de desconsiderar lo anteriormente señalado, se corre el riesgo de adulterar el espacio, reduciendo sus funciones técnicas de sobrevivir. El análisis se realizó a través de la modelación cartográfica y geoestadística. Se trazó un comparativo de la distritación en ambos años, se generaron análisis de concentración-dispersión espacial, a través de la unión entre cartografía y el Censo de Población y Vivienda 2010; se modelo una matriz de correlación con las variables de residencia; $y$, se generaron modelos lineales que nos permitieron establecer proyecciones entre los aspectos que determinan, a escala nacional, la votación por cada uno de los tres principales partidos políticos: Partido Acción Nacional (PAN), Partido Revolucionario Institucional (PRI) y Partido de la Revolución Democrática (PRD).
\end{abstract}

Palabras clave: distritos, espacio, $r$ statistic software, geoestadística, ordenamiento del território.

\section{Resumo}

Notas territoriais e estatísticas no software estatístico R: entender os distritos eleitorais no México

México é dividido em 32 estados que compõem a Federação. Os 300 distritos que constituem parte do poder Legislativo estão localizados em todos os estados. Nos anos de 1996 e 2005, reconfigurou-se a sua forma, o que teve impacto em sua estrutura. Interessa mostrar que a redistritación não é uma questão de forma, sem conteúdo, uma vez que uma alteração da forma do espaço é uma alteração na essência, ou seja, é uma ação técnica que impacta na estrutura da geografia de ações sociais e a paisagem dos recursos, portanto, é uma ação improdutiva que impacta valorizando ou desvalorizando o espaço das ações produtivas. Em caso de desconsiderar o anteriormente mencionado, corre-se o risco de adulterar o espaço, reduzindo suas funções técnicas de sobreviver. A análise foi feita através da modelação cartográfica e geoestatística. Traçou-se um comparativo entre os distritos em ambos anos, foram geradas análise de concentração e

\footnotetext{
${ }^{1}$ Doutor em geografia pela Universidade Estadual Paulista do Brasil e docente do Departamento de Ciências Sociais, Universidad Autónoma Metropolitana, Unidad Cuajimalpa. E-mail: luna@correo.cua.uam.mx
} 
dispersão espacial, através da união entre a cartografia e o Recenseamento da População e Habitação de 2010; é modelo de uma matriz de correlação com as variáveis de residência; e, foram gerados modelos lineares que nos permitiram estabelecer projeções entre os aspectos que determinam, a nível nacional, a votação para cada um dos três principais partidos políticos: Partido Acción Nacional (PAN), o Partido Revolucionario Institucional (PRI) e o Partido de la Revolución Democrática (PRD).

Palavras-chave: distritos, espaço, $r$ statistic software, geoestadística, ordenamento do território.

\section{Summary}

Territorial and statistics notes in software $R$ : understand the electoral districts in México

Mexico is divided into 32 states that make up the Federation. In the 32 states are located in the $\mathbf{3 0 0}$ districts that are part of the Legislative power. In the years 1996 and 2005 is reconfigured its way impacted on its structure. Want to show that redistricting is not a matter of form without content, since a modification in the shape of the space is a modification in the essence, that is to say, it is a technical action that impacts on the structure of the geography of social action and the landscape of resources, therefore, is a non-productive action that impacts valuing or devalue the space of the productive actions. In case of disregarding the above-mentioned, you run the risk of adulterating the space, reducing their technical functions to survive. The analysis was performed through the modeling, mapping and geostatistics. We drew up a comparative distritación in both years, we generated analysis about concentration and dispersion spatial, through the union between cartography and the Census of Population and Housing 2010; model a correlation matrix with the variables of residence; and, generated linear models that allowed us to establish projections among the aspects that determine, at national level, the voting for each of the three main political parties: Partido Acción Nacional (PAN), Partido Revolucionario Institucional (PRI) and Partido de la Revolución Democrática (PRD).

Keywords: districts, space, $r$ statistic software, geoestadística, spatial planning. 


\section{Introducción}

En el año de 1990, en México, se creó el Instituto Federal Electoral (IFE) ${ }^{2}$ con dos motivaciones fundamentales: 1) integrar el Registro Federal de Electores; y, 2) actualizar la cartografía electoral del país, tipificada como: Entidad, Distrito Electoral Federal, Municipio y Sección electoral (IFE, 2005b; 2015). Ambas acciones con el fin de encausar las elecciones federales del año 1991.

Antes de 1990, se contaba con información integrada de los distritos y secciones electorales sin métrica ni georreferencia precisa, desactualizada en torno a los cambios en las zonas urbanas y rurales, además, se tenía la incertidumbre de incluir todas las localidades (IFE, 2005a).

Tanto en el año de 1996 como en el año 2005, un año antes de las elecciones de 1997 y 2006, respectivamente, se generaron procesos de redistritación, considerando los datos poblacionales del Censo de Población y Vivienda 1990 y 2000, que genera el Instituto Nacional de Estadística Geografía e Informática (INEGI). Hacia la preservación de los límites municipales, conformación de la población indígena, accesibilidad en la forma de los distritos y tiempo de desplazamiento entre las localidades de cada distrito (IFE, 2005a; 2005b; COLLHURTADO, 2008).

El último proceso de distritación se realizó en el año 2017, siguiendo los mismos pasos que los años anteriores: a) Con base en el Censo de Población más reciente, distribuir la población en 300 polígonos que abarcan toda la República Mexicana; b) diseño de un algoritmo de optimización para un mapa preliminar para cada estado; c) dos rondas de modificaciones por parte de los partidos políticos, d) el Comité Técnico selecciona la propuesta final y somete a consideración del Consejo General para su aprobación.

En el presente artículo realizaremos un análisis territorial de las redistritaciones electorales, con base en la aplicación de test estadísticos en $\mathrm{R}$ y de un banco de datos construido con las variables del Censo de Población y Vivienda 2010. Entre los estadísticos utilizados se encuentra la correlación, modelo de regresión y medidas de tendencia central.

\footnotetext{
${ }^{2}$ Cambiando de nombre tras fraudes electorales como el del año 2006, se realizaron súper gastos en la compra de voto y se inflaron casillas donde no había vigilancia de los tres principales partidos, justificando el triunfo "haiga sido como haiga sido", denominado, ahora, Instituto Nacional Electoral (INE).
} 
Asimismo, se realiza un análisis cartográfico de concentración y dispersión emigratoria, poblacional y cantidad de distritos por cada uno de los estados que componen la federación.

La investigación muestra, a partir del subtítulo número 2, las características de la herramienta tecnológica $R$, instrumento que permitió observar y explicar los datos. En el apartado 3, se presenta el método de investigación. El bloque 4, contiene los resultados de la prueba de hipótesis, los test y la regresión. En el bloque 5, se discute con diversos autores la pertinencia técnica, ¿En realidad resuelve el problema de equidad que se busca en la representación de los trescientos distritos electorales federales? Finalmente, la sección 5, recopila los principales datos que nos permiten concluir con el análisis.

\section{Características actuales de $\mathbf{R}$}

El software R es una poderosa e innovadora herramienta de programación estadística y gráfica. Actualmente, está siendo integrada al análisis de datos por parte de los investigadores sociales. La experiencia y la práctica han demostrado la idoneidad del programa, para el análisis de redes migratorias, movilidad urbana y análisis de datos electorales. Asimismo, por más de un año ininterrumpido, con dicha herramienta se imparten cursos sobre problemas actuales para los alumnos de la Licenciatura en Políticas Públicas y de la Licenciatura en Estudios Socioterritoriales de la Universidad Autónoma Metropolitana, México. El programa es libre, tiene a su disposición un sistema de recursos que lo complementan, hay más de 2,000 complementos disponibles $R$ (TEETOR, 2011). Compite con todos los paquetes estadísticos comerciales.

$R$ no es un paquete simple de utilizar. Diversos autores que han realizado manuales y desarrollado la herramienta para su uso, concuerdan en la dificultad inicial para efectuar operaciones sencillas (TEETOR, 2011; ADLER, 2010; AHO, 2015; BEAUJEAN, 2014; PATHAK, 2014; BILDER and LOUGHIN, 2015; BIVAND, PEBESMA and GÓMEZ RUBIO, 2013; GAETAN and GUYON, 2010; ARNOLD and TILTON, 2015). Las operaciones sencillas parecen fáciles una vez que se sabe cómo realizarlas, no obstante el cómo puede resultar exasperante.

El lenguaje R se basa en el antiguo idioma S, desarrollado en los Laboratorios Bell. Estructura desarrollada primeramente en el MIT. Ihaka y Gentleman utilizaron el nombre de R 
para reconocer la influencia de $S$, debido a que $R$ y $S$ son yuxtapuestos en el alfabeto (TEETOR, 2011).

El paquete R no tiene garantías, puesto que es libre. Su marco de derecho de autor es GNU, acrónico del inglés no es Unix. La Licencia Pública General (PGL) permite a los usuarios compartir y cambiar las funciones y complementos de R.

Beaujean (2014) reduce el estrés para los inexpertos, al señalar que el programa $R$ es de mayor flexibilidad y facilidad que otros programas. Por ejemplo: al ejecutar un modelo de regresión múltiple o el uso de diferentes conjuntos de predicción, se dispone de la oportunidad de utilizar los primeros resultados para subsecuentes análisis. Por otra parte, si el análisis se deja pendiente por algunos días en el historial se puede observar las secuencias utilizadas para obtener los resultados anteriores.

RStudio muestra sus coeficientes y resultados en una ventana. En lugar de abrir muchas, en la misma pantalla, con ayuda de RStudio, se puede contar con cuatro secciones: 1) Note Block; 2) Environment-History; 3) Output; 4) Files-Plots-Packages-Help-Viewer. Se puede alimentar la sintaxis, copiándolas en posteriores análisis o bien obtener nuevamente los resultados de ésta. A continuación se muestra en la Tabla 1, la sintaxis básica para el uso del software.

Tabla 1. Algunos comandos básicos para el uso del software

\begin{tabular}{|c|c|c|}
\hline Concepto & Descripción & Sintaxis \\
\hline Comentario & $\begin{array}{l}\text { Se utiliza para realizar anotaciones que permiten ahorrar tiempo y } \\
\text { esfuerzo }\end{array}$ & \# \\
\hline Asignar & $\begin{array}{l}\text { Es el símbolo de menor que, seguido de un guion medio. Asigna } \\
\text { un operador, equivale a utilizar }=\text {, pero es mejor reservar el signo } \\
\text { de igual para definir valores en los argumentos. Asigna lo que está } \\
\text { a la derecha de la flecha para el objeto a la izquierda de la flecha. }\end{array}$ & $<-$ \\
\hline Concatenar & $\begin{array}{l}\text { La función concatenar, conjunta los argumentos dentro de una } \\
\text { función. Usando } c() \text { en conjunto con }(<-) \text { asigna la concatenación } \\
\text { de objetos dentro de un nuevo objeto. Por ejemplo: } \\
\text { Fibonacci<-c(1,1,2,3,5,8,13,21,...) }\end{array}$ & $c()$ \\
\hline Ayuda & $\begin{array}{l}\text { La función ayuda (help) regresa información sobre una función. Se } \\
\text { puede escribir la función o el símbolo de interrogación. Ejemplo: } \\
\text { help(mean) } \\
\text { ?mean }\end{array}$ & $\begin{array}{l}\text { help }() \\
? \ldots\end{array}$ \\
\hline Ejemplo & $\begin{array}{l}\text { Se utiliza para mostrar un ejemplo de la función. Ejemplo: } \\
\text { example(mean) }\end{array}$ & example() \\
\hline Modelo lineal & $\begin{array}{l}\text { Modelo estadístico que mide la varianza }(r) \text { entre una variable } \\
\text { dependiente y una o más variables independientes. }\end{array}$ & $\operatorname{lm}()$ \\
\hline Base de datos & La función read, permite montar una base de datos separada por & read.csv() \\
\hline
\end{tabular}




\begin{tabular}{|c|c|c|}
\hline & $\begin{array}{l}\text { comas. Modificando la extensión se pueden cargar bases de datos } \\
\text { en diferentes formatos, por ejemplo: texto (.txt), excel (.xlsx), SPSS } \\
\text { (.spss), DBF (.dbf). }\end{array}$ & \\
\hline $\begin{array}{l}\text { Instalar } \\
\text { complemento }\end{array}$ & $\begin{array}{l}\text { Función que sirve para instalar complementos y realizar análisis } \\
\text { estadísticos, de redes y cartográficos con mayor desarrollo y } \\
\text { precisión. }\end{array}$ & install.packages() \\
\hline Correlación & $\begin{array}{l}\text { La función correlación calcula la varianza de } x \text {, y. El coerifiente de } \\
\text { correlación es una medida numérica de relacionamiento } \\
\text { estadístico entre dos variables, varía entre }-1 \text { a }+1 \text {, donde }+1 \\
\text { indica la máxima correlación positiva, } 0 \text { significa que no hay } \\
\text { correlación entre las variables y }-1 \text { indica máxima correlación } \\
\text { negativa. }\end{array}$ & $\operatorname{cor}()$ \\
\hline Librería & $\begin{array}{l}\text { Función para abrir la biblioteca de un complemento. Cada que } \\
\text { hacemos uso de dicho complemento, debemos llamar primero la } \\
\text { biblioteca a la cual éste pertenece. }\end{array}$ & library() \\
\hline
\end{tabular}

Elaboración propia con base en Teetor (2011) y Cotton (2013). Actualmente, se puede montar una base de datos sin necesidad de un vector. Es cuestión de seguir la rutina de Import Dataset localizada en la sección Environment de RStudio.

\section{Método}

Basados en el principio de equidad social y partidista sobre el que se orientan los esfuerzos técnicos en las redistritaciones del territorio nacional para que todos los individuos estén representados en el poder legislativo, se van a testar dos hipótesis, $h_{1}$ y $h_{0}$, la primera cuestiona el objetivo de la redistritación: que no hay tal principio de equidad social y partidista, la soluciones técnicas, se amparan en el supuesto objetivo y positivo expuesto por la institución a cargo de ésta, para resarcir los espacios que la pluralidad democrática arrebatada al grupo hegemónico que busca seguir manteniéndose en el poder; la segunda hipótesis concuerda con los objetivos del procedimiento, señala que todos los esfuerzos técnicos, efectivamente, de una manera neutra, sin inclinaciones de cualquier tipo, están dirigidos a garantizar la equidad social y partidista.

$h_{1}$ : Los estados con mayor número de distritos en el año 1996 tuvieron una modelación más fragmentada o compactada del territorio, aumentando o reduciendo el número de distritos en los año 2005 y 2017. Eliminando el principio de equidad social, pluralidad democrática y partidista.

$h_{0}$ : Los estados con mayor número de distritos en el año 1996 no tuvieron una modelación más fragmentada o compactada del territorio, aumentado o reduciendo el 
número de distritos en el año 2005. Buscando el principio de equidad social, pluralidad democrática y partidista.

En el ejercicio de gobernar, el Estado precisa fragmentar el territorio, tal como se muestra en las distritaciones a través de la Tabla 2. El sistema de acciones económicas, productivas del espacio, en sus dos formas, dominio y empoderamiento, permite reconstituir los fragmentos en la totalidad, resultado de estos dos actos es el valor o disvalor del espacio (SANTOS, 2000; LUNA, 2014; LUNA, 2016a; LUNA, 2017, Hirsch, 2001). “El valor del espacio no es independiente de las acciones que es susceptible de acoger" (SANTOS, 2000. p. 74).

El análisis geoestadístico se realizó por medio del programa estadístico $\mathrm{R}$, previa construcción del banco de datos unido a la cartografía. La sintaxis principal y prueba de un mapa de salida se muestran a continuación.

install.package(“tmap)

ibrary(tmap)

library(maptools)

Fede<-readShapeSpatial("C:/LASTense/Plataforma/Partidos y Elecciones intermedias/DistritosZM96-04/mapavar iables/09VARIABLESOCIALES.shp")

(Entre comillas se escribe la localización, dentro del ordenador, del shape)

\section{Resultados}

El fenómeno de la redistritación se complica aún más con las candidaturas independientes, que adquieren legitimidad en el año 2012, con la reforma política, mismas que se ponen en práctica en el año 2015. Se adhieren grupos sociales a la disputa del territorio, sin embargo, el fortalecimiento de los grupos no se produce con la fragmentación y agregación de nuevas candidaturas.

Se está disputando la producción del territorio a través de los distritos electorales. Las instituciones que orientan los procesos electorales no propician el arraigo territorial de los partidos. Los grupos sociales no tienen un proyecto a largo plazo para desarrollarse en procesos de consolidación de sujetos sociales con autonomía y en rutas de empoderamiento, guían su conducta sobre la base del carácter receptivo, esperando que los partidos políticos ofrezcan recursos y beneficios materiales (FROMM y MACCOBY, 2007). Al mismo tiempo hay que sumarle a varios grupos sociales que conforman: "todos contra el candidato efectivamente plural" que ha sido ensayado en las elecciones del año 2017 con la candidata al Estado de México. 
La redistribución de los 300 distritos de 1996 al año 2017, se dirige por el sendero del prolegómeno que señala que no se puede otorgar un beneficio sin afectar a otro (LUNA, 2016a). Estado de México gana durante el proceso cinco distritos, mientras que la Ciudad de México y Veracruz reducen seis y tres distritos, respectivamente. Cabe señalar que hay otros estados que pierden un distrito y otros que lo ganan, pero los mencionados son los más representativos del fenómeno. Ver Tabla 2.

El caso del Estado de México, en el año 2017, Ecatepec, el municipio más poblado del país aumentó de 4 a 6 distritos; el municipio contiguo, Nezahualcoyotl se compactó de 5 a 3 , Naucalpan creció de 2 a 3, entre otros municipios del Estado de México, donde el PRI ha volcado todas sus habilidades para mantener su representación. Ver Tabla 2.

Tabla 2. Distritos por entidad, 1996-2017.

\begin{tabular}{|c|c|c|c|c|}
\hline Clave & Entidad & $\begin{array}{c}\text { Distritos } \\
1996\end{array}$ & $\begin{array}{c}\text { Distritos } \\
2005\end{array}$ & $\begin{array}{c}\text { Distritos } \\
2017\end{array}$ \\
\hline 1 & Aguascalientes & 3 & 3 & 3 \\
\hline 2 & Baja California N & 6 & 8 & 8 \\
\hline 3 & Baja California S & 2 & 2 & 2 \\
\hline 4 & Campeche & 2 & 2 & 2 \\
\hline 5 & Coahuila & 7 & 7 & 7 \\
\hline 6 & Colima & 2 & 2 & 2 \\
\hline 7 & Chiapas & 12 & 12 & 13 \\
\hline 8 & Chihuahua & 9 & 9 & 9 \\
\hline 9 & Ciudad de México & 30 & 27 & 24 \\
\hline 10 & Durango & 5 & 4 & 4 \\
\hline 11 & Guanajuato & 15 & 14 & 15 \\
\hline 12 & Guerrero & 10 & 9 & 9 \\
\hline 13 & Hidalgo & 7 & 7 & 7 \\
\hline 14 & Jalisco & 19 & 19 & 20 \\
\hline 15 & México & 36 & 40 & 41 \\
\hline 16 & Michoacán & 13 & 12 & 12 \\
\hline 17 & Morelos & 4 & 5 & 5 \\
\hline 18 & Nayarit & 3 & 3 & 3 \\
\hline 19 & Nvo. León & 11 & 12 & 12 \\
\hline 20 & Oaxaca & 11 & 11 & 10 \\
\hline 21 & Puebla & 15 & 16 & 15 \\
\hline 22 & Querétaro & 4 & 4 & 5 \\
\hline 23 & Quintana Roo & 2 & 3 & 4 \\
\hline 24 & San Luis & 7 & 7 & 7 \\
\hline 25 & Sinaloa & 8 & 8 & 7 \\
\hline
\end{tabular}




\begin{tabular}{|c|c|c|c|c|}
\hline 26 & Sonora & 7 & 7 & 7 \\
\hline 27 & Tabasco & 6 & 6 & 6 \\
\hline 28 & Tamaulipas & 8 & 8 & 9 \\
\hline 29 & Tlaxcala & 3 & 3 & 3 \\
\hline 30 & Veracruz & 23 & 21 & 20 \\
\hline 31 & Yucatán & 5 & 5 & 5 \\
\hline \multirow[t]{2}{*}{32} & Zacatecas & 5 & 4 & 4 \\
\hline & Total general & 300 & 300 & 300 \\
\hline
\end{tabular}

Elaboración propia con base en IFE (2005a; 2017).

Los procesos de distritación provocan un sesgo para favorecer la votación a determinado partido. Como señala Lacoste (1977, p.5): “La geografía es un arma para la guerra [...] Un instrumento de fuerza para los que ostentan el poder".

La geografía es, un saber estratégico estrechamente unido a un conjunto de prácticas políticas y militares, y son dichas prácticas las que exigen la recopilación articulada de unas informaciones extremadamente variadas y a primera vista heterogéneas (LACOSTE, 1977. p. 5).

El efecto salamandra es uno de los riesgos más conocidos en la formación de distritos. A través de éste fenómeno se genera una representación parlamentaria separada de la decisión de los electores. Se manipula concientemente el trazo de la escala geolectoral para favorecer o reducir a un grupo. Su origen se remonta al año 1912, cuando el gobernador de Massachussets, USA, Elbridge Gerry, modeló un polígono con apariencia de salamandra, que le aseguraba el triunfo (IFE, 2005).

A continuación se muestra, en la Figura 1, un polígono por cada uno de los 32 estados, representando la población. Los polígonos integran el trazo de los distritos. Los distritos se representan en color azul, yendo del tono más débil al más fuerte, dependiendo de la cantidad de población que alberga la escala geoelectoral, cuando el tono es débil significa menor población que cuando es más fuerte. Cabe mencionar que en la Figura $\mathbf{2}$ se muestra el mismo tema, pero con los 32 polígonos unidos, con la finalidad que se contraste de manera local y federal, la composición de población por distrito.

tm_shape(Fede) +

+ tm_fill("DISTRITO", legend.show = FALSE) +

+ tm_facets("ENTIDAD") 
Figura 1. Población por distrito a escala estatal, 2005.
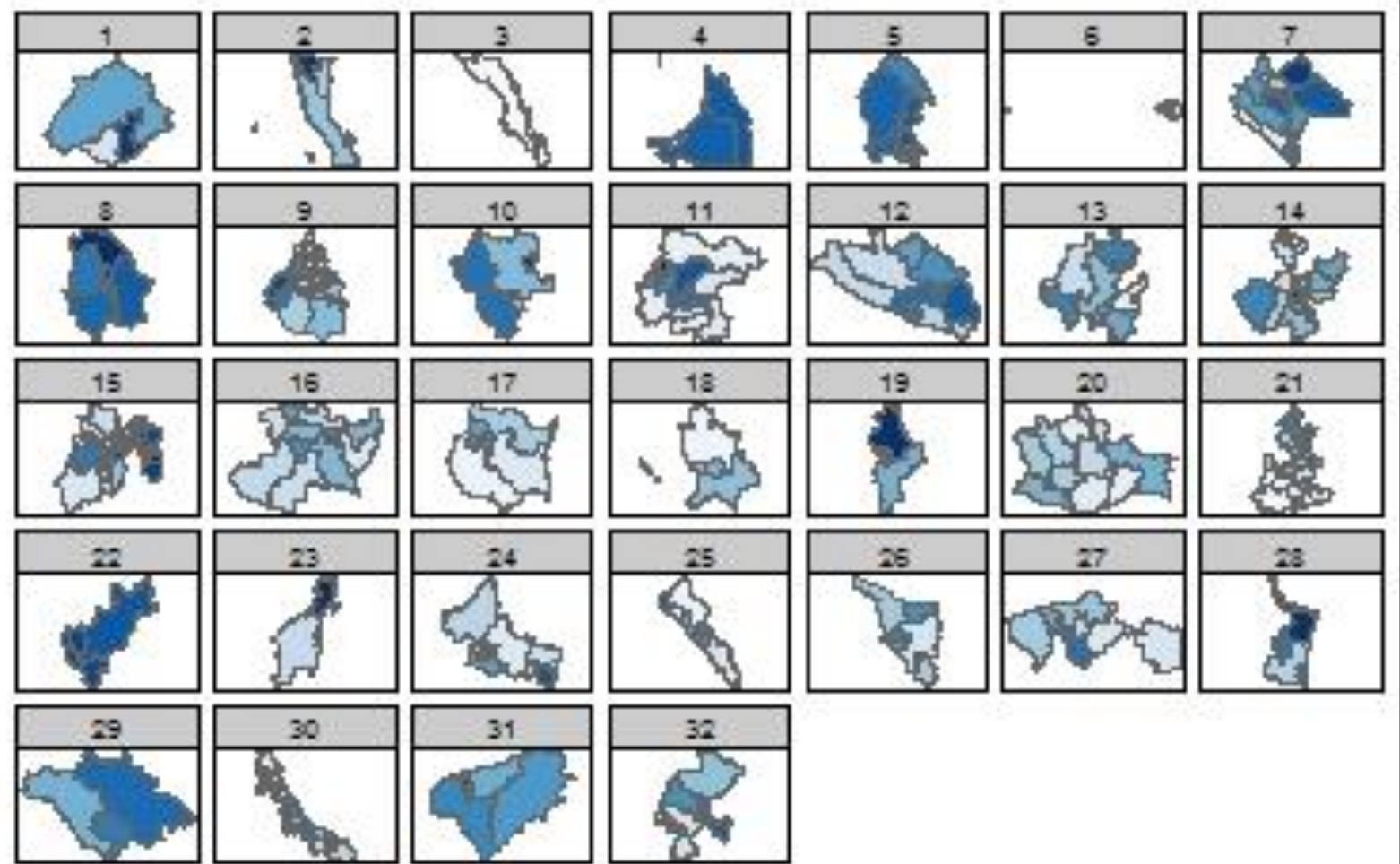

Fuente: Elaboración propia con base en INEGI (2010) y Arnold and Tilton (2015).

Con respecto a los estados que tienen mayor número de distritos y la relación con la mayor concentración de población, podemos observar que el Estado de México y la Ciudad de México tienen una dinámica particular, no obstante el menú de opciones se expande a otros estados que se localizan en las zonas francas: Ciudad Juárez-Chihuahua, Tijuana-Baja California, Matamoros-Tamaulipas, Isla Mujeres-Cancún y Cozumel-Quintana Roo. Es en estas zonas donde está concentrada la industria maquiladora y de turismo, en el año 2014 reporté “906 Maquiladoras en Baja California, 402 en Chihuahua y 339 en Tamaulipas" (LUNA, 2014, p 65). Cabe mencionar que a mayor territorio no significa más poder para la población, sin embargo, en los distritos con mayor población es donde se tiene mayor cantidad de recursos para generar riqueza, como por ejemplo: la fuerza de trabajo y los recursos naturales. Los distritos con mayor población infieren el factor salamandra, lo que se combina con un sistema que impacta a los demás distritos, pues como ya se mencionó, el prolegómeno es que no se puede otorgar poder sin afectar a otros. 
En cuanto a la concentración de población podemos observar que en las zonas fronterizas se encuentran los distritos con mayor concentración de población. Los rangos establecidos fueron 4, del tono más sutil al más fuerte:

rango uno, mínimo 243.902, máximo 309.106;

rango dos, mínimo 309.107, máximo 374.311;

rango tres, mínimo 374.312, máximo 439.516; y,

rango cuatro, mínimo 439.517, máximo 504.722. Ver Figura 2.

Figura 2. Población por distrito a escala nacional, 2010.

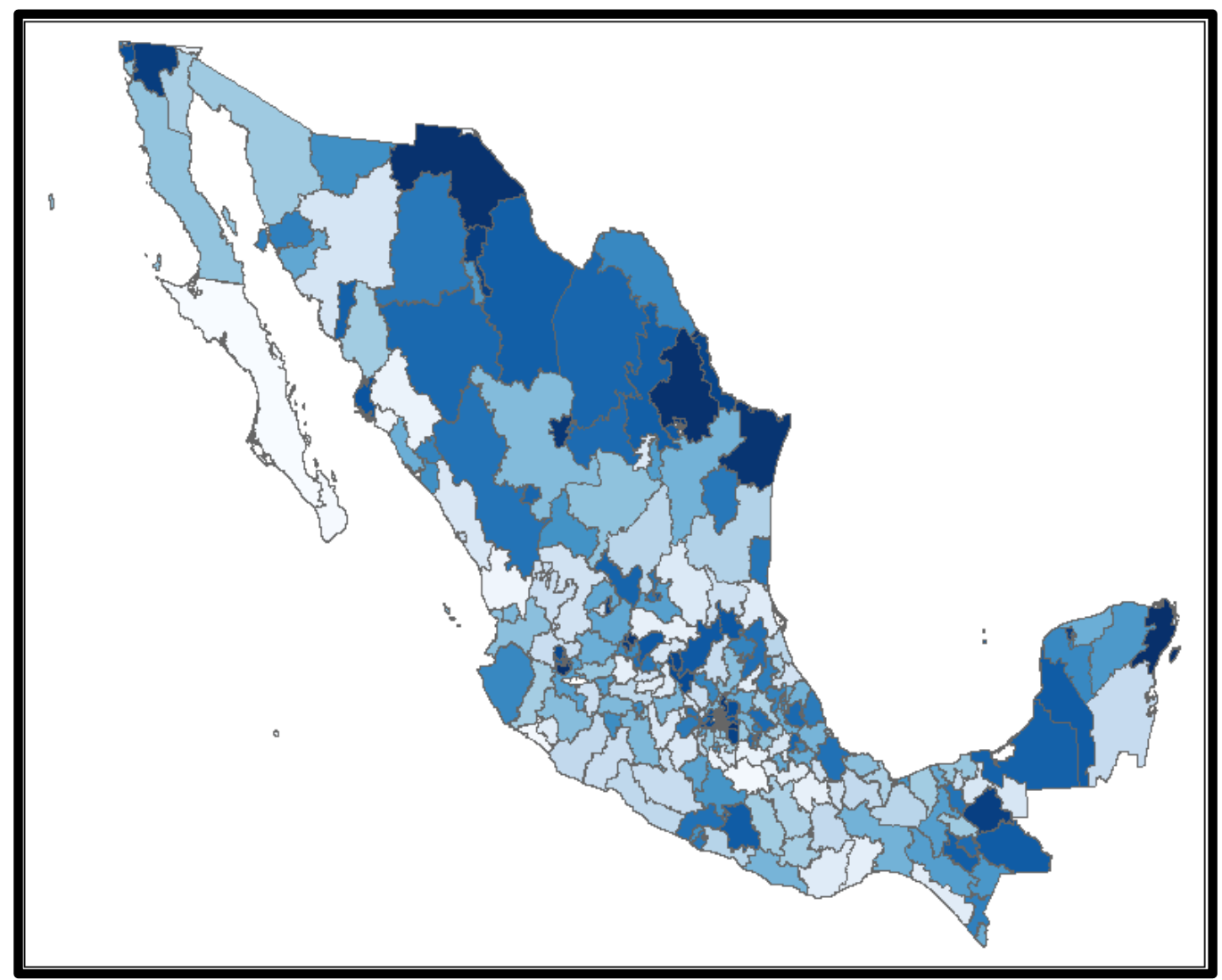

Fuente: Elaboración propia con base en INEGI (2010).

Francisco de Oliveira (1982) analiza que las condiciones de posibilidad para la planificación del territorio se sostienen por las relaciones sociales de producción: el cálculo económico. La ecuación del cálculo económico se formula por el contenido de valor de las mercancías, entre ellas la fuerza de trabajo, factor que es exponencial por la reiteración de los intercambios y de la circularidad del capital, más la constante, ese equivalente general.

Dicha ecuación al fragmentar la producción a través de la división de la fuerza de trabajo lo que genera es un desconocimiento geográfico en la población (OLIVEIRA, 1982; 
LACOSTE, 1977). No cabe duda que la formulación técnica que agrega la variable demográfica, los límites municipales, población indígena, forma de los distritos y tiempo de desplazamiento entre localidades sirve a los propósitos del cálculo económico y no a la representación de la población y sus condiciones materiales, para ser escuchados en el poder Legislativo (LUNA, 2016b; LUNA, 2017). Reduciendo el poder de la población y su conocimiento geográfico.

Se generaron predicciones a través del modelo de regresión lineal. Una de las predicciones que se realizó fue una relación entre los principales partidos políticos y los rangos de edad de los votantes. El modelo supone que en el año 2012, el $41 \%$ de la participación orientada al Partido Acción Nacional está determinado por el rango de edad de los participantes, conformado por jóvenes de 20 a 24 años de edad y adultos de dos rangos, 50 a 54 años y 55 a 59 años de edad. Del año 2009 al 2012 se presentó un aumento en el coeficiente de varianza, casi el doble, debido a que en el año 2009 el porcentaje fue de $21 \%$.

En relación con el PRI en el año 2012, el modelo lineal señala que la participación por edad está condicionada en un 39\%, siendo los rangos de edad que más le favorecen son de 15 a 19 años y de 55 a 59 años. Para el PRD se presenta el $47 \%$ de su votación está relacionada con la edad, siendo el rango de población cautiva el de 25 a 29 años de edad.

El modelo se corrió para cada uno de los principales partidos en el software $R$, a través de un vector que conjunta la variable dependiente (VD), votación para el partido en el año señalado, frente a las variables independientes (VI), todos los rangos de edad que agrupa el Censo de Población y Vivienda 2010, rangos de cinco años. Datos observados en la siguiente sintaxis de la Tabla 3.

Tabla 3. Sintaxis del modelo lineal múltiple

Nombre del vector<-Im(VD VI1+VI2+VI3+VI4..., data=Nombre del banco de datos utilizado) Elaboración propia.

Se generó una matriz de correlación emigratoria interna, es decir, desplazamientos entre las 32 entidades federativas. Los posible valores de la varianza son de -1 a 1 , los valores positivos se representan en la Matriz 1 con el color azul, no hay presencia de valores negativos, aunque hay celdas que se representan como valor 0. 
Matriz 1. Correlación de emigración interna: México, 2010.

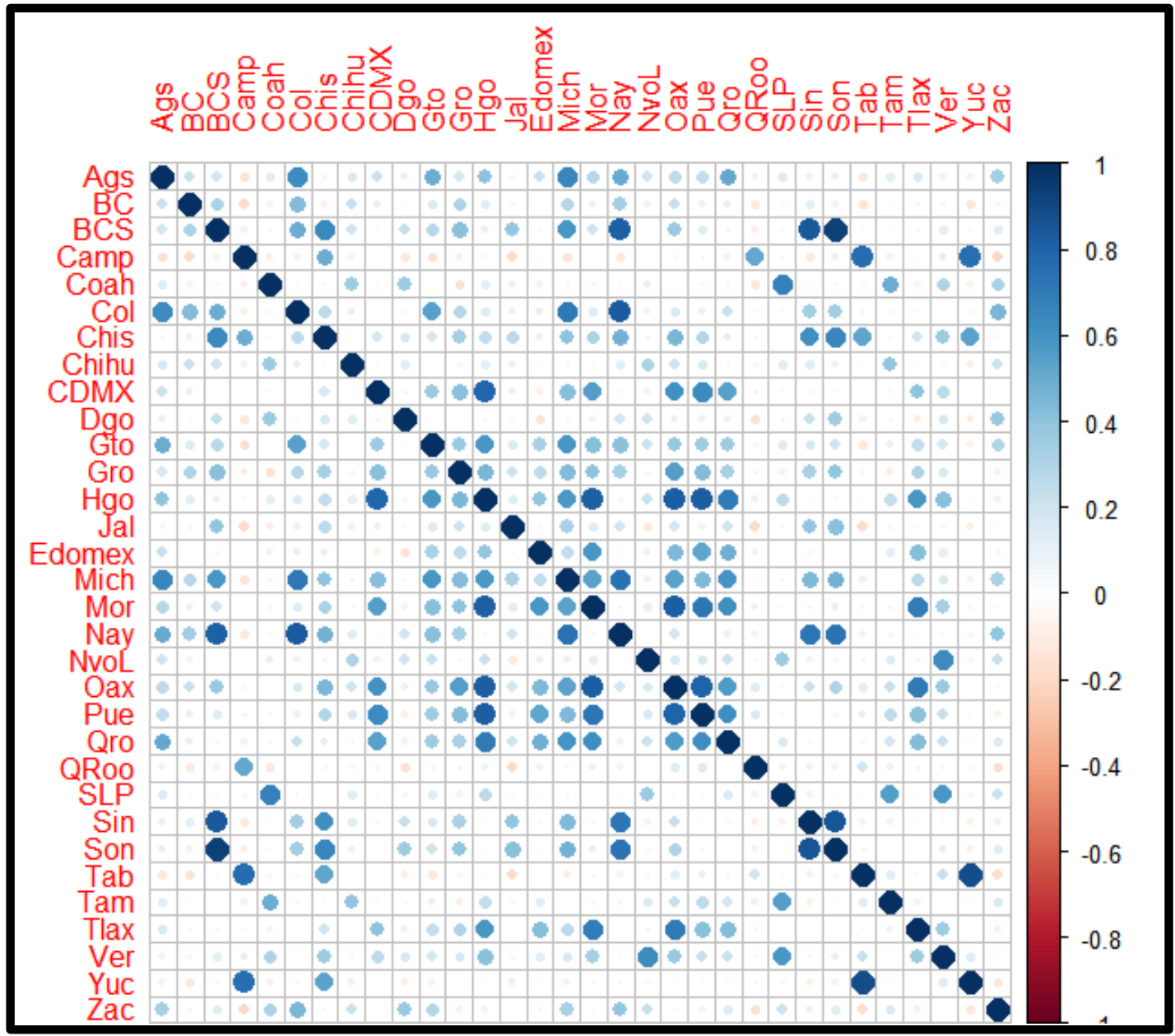

Fuente: Elaboración propia con base en INEGI (2010).

La matriz se generó con una tabla de $32 \times 32$ con la variable residencia, véase Tabla 4. Es imposible generar la matriz a escala de distrito, porque no se encuentran las variables desagregadas hasta ese nivel geográfico. Para este análisis se instaló el complemento: corrplot.

Tabla 4. Sintaxis de la matriz de correlación de emigración interna Nombre del vector<-corrplot(Nombre de la matriz[c("nombres de renglones"), c("nombres de las variables entre comillas")], use= "all.obs".

Elaboración propia.

La planificación, en este caso de los distritos electorales, tiene esa peculiaridad que señala Oliveira (1982, p. 13), sirve y subraya "sin sombra de duda" como una "forma técnica de la división del trabajo" [...] "Técnica de la división del trabajo improductivo que gobierna al 
trabajo productivo". Se elaboró una cartografía que muestra las interacciones migratorias, evidenciando la tendencia emigratoria del norte hacia el sur, véase Figura 5.

Figura 5. Red de emigración interna

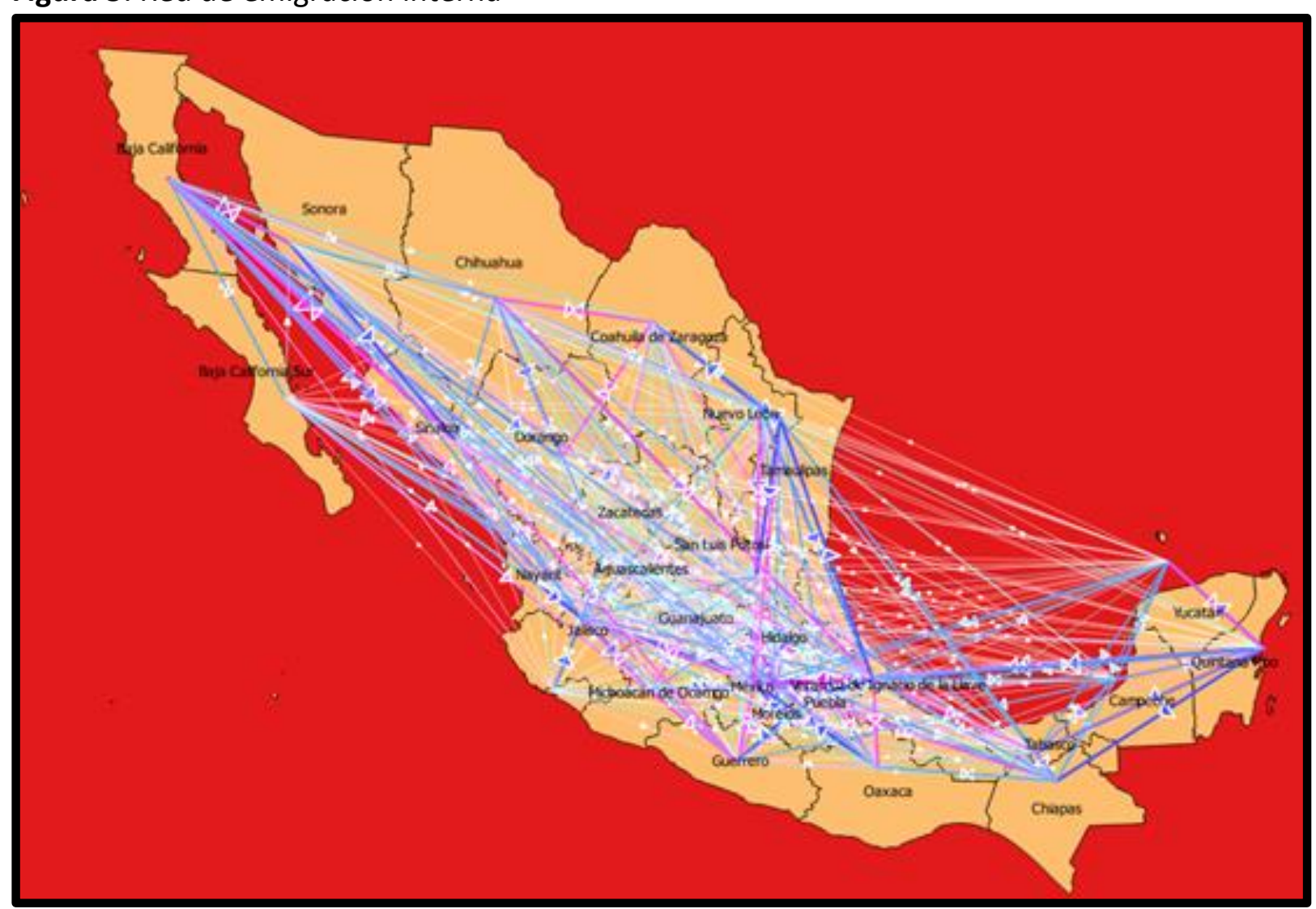

Fonte: Elaboración propia con base en INEGI (2010).

Es evidente que la planificación en el modo de producción capitalista, como el de México, es una manera de racionalización de la reproducción ampliada del capital. "El Estado trasforma una parte de los impuestos para ordenar el espacio y ponerlo a disposición del capital" (OLIVEIRA, 1982, p.24). Surge una nueva relación que muestra que no es sólo el Estado el que planifica el plan maestro del capitalismo, sino que el capitalismo planea la planificación (TAUSSIG, 2015). Los distritos que no disponen de un nombre para dificultar su dominio por parte de sus habitantes, sino de una clave compuesta por la clave del estado, formada por dos dígitos y dos dígitos del distrito, tienen más que ver con la forma económica y política y una forma particular de dominio y resistencia que con los supuestos cálculos de movilidad y accesibilidad que dieron pie a su conformación en el año 1996 y su reestructuración en los años 2005 y 2017. 
Finalmente, se realizó por partido, PRI, PAN y PRD, un modelo de regresión con el fin de interpretar las características culturales, del lugar y de la vivienda que favorecen la votación por partido. Las variables elegidas fueron: hablante de lengua indígena, sexo, seguridad social en el Imss, Issste, Pemex, Seguro popular, Institución privada, población alfabeta, población analfabeta, tamaño de localidad, disposición de energía eléctrica, televisión, refrigerador, lavadora y computadora en la vivienda, y, por último, tipo de hogar.

El modelo del PAN para el año 2015, véase Tabla 5, señala que el 51.3\% de la votación por el partido se explica por el conjunto de variables elegidas. Sobresaliendo que el perfil de sus electores tienen Imss, son analfabetas y disponen de energía eléctrica y televisión. Con un nivel de confianza del $99 \%$ como lo marcan los asteriscos que se ubican a la derecha de los coeficientes que se muestran a continuación.

El modelo del PRI para el año 2015, véase Tabla 6, señala que el 43.7\% de la votación por el partido se explica por el conjunto de variables elegidas. Sobresaliendo que el perfil de sus electores tienen Imss, no disponen de energía eléctrica y la composición del hogar es ampliado (formados por un hogar nuclear más otros parientes). Con un nivel de confianza del 99\% como lo marcan los asteriscos que se ubican a la derecha de los coeficientes que se muestran a continuación.

El modelo del PRD para el año 2015, véase Tabla 7, señala que el 33.7\% de la votación por el partido se explica por el conjunto de variables elegidas. Sobresaliendo que el perfil de sus electores tiene Imss, dispone de refrigerador y el tipo de hogar es compuesto (constituido por un hogar nuclear o ampliado, más personas sin parentesco con el jefe del hogar). Con un nivel de confianza del $99 \%$ como lo marcan los asteriscos que se ubican a la derecha de los coeficientes que se muestran a continuación.

Tabla 5. sintaxis del modelo de regresión PAN

Call: $\operatorname{Im}($ formula $=$ PAN15 $\sim$ hablein $1+$ sexo_ $1+$ sexo_ $2+$ imss $1+$ issste $1+$ 
pemex1 + segu_pop1 + inst_pri1 + alfabet1 + alfabet $2+$ tam_loc01 + tam_loc02 + tam_loc03 + tam_loc04 + tam_loc05 + tam_loc06 + tam_loc07 + tam_loc08 + tam_loc09 + tam_loc10 + tam_loc11 + tam_loc12 + tam_loc13 + tam_loc14 + tam_loc15 + dis_elec1 + dis_elec $2+$ dis_elec $9+$ dis_tele $1+$ dis_refr $1+$ dis_lava $1+$ dis_comp1 + ticlahog11 + ticlahog12 + ticlahog13, data = basame)

Elaboración propia

Residuals:

Min 1Q Median 3Q Max

$\begin{array}{lllll}-33094 & -8517 & -521 & 6881 & 43650\end{array}$

Coefficients:

Estimate Std. Error $\mathrm{t}$ value $\operatorname{Pr}(>|\mathrm{t}|)$

(Intercept) $1.360 \mathrm{e}+04 \quad 8.865 \mathrm{e}+03 \quad 1.5340 .126264$

hablein1 $1.285 \mathrm{e}-01 \quad 4.943 \mathrm{e}-02 \quad 2.6000 .009857 * *$

sexo_1 -1.279e+00 4.037e-01 $-3.1680 .001717^{* *}$

sexo_2 -8.699e-01 3.882e-01 $-2.2410 .025879 *$

imss1 2.787e-01 4.414e-02 $6.3131 .15 \mathrm{e}-09 * * *$

issste1 -2.410e-01 $1.076 \mathrm{e}-01-2.2390 .025978$ *

pemex1 -3.412e-02 1.419e-01 -0.2400 .810157$

segu_pop1 7.621e-02 3.880e-02 1.9640 .050545 . inst_pri1 $\quad 4.814 \mathrm{e}-01 \quad 1.798 \mathrm{e}-01 \quad 2.6770 .007902 * *$ alfabet1 $\quad 1.278 \mathrm{e}+00 \quad 3.986 \mathrm{e}-01 \quad 3.2060 .001509 * *$ alfabet2 $1.600 \mathrm{e}+00 \quad 4.079 \mathrm{e}-01 \quad 3.9230 .000111 * * *$ tam_loc01 $1.239 \mathrm{e}-01 \quad 4.185 \mathrm{e}-01 \quad 0.2960 .767487$ tam_loc02 -2.328e-01 7.119e-01 -0.327 0.743942 tam_loc03 3.893e-01 $1.950 \mathrm{e}-01 \quad 1.9960 .046925$ * tam_loc04 $1.789 \mathrm{e}-01 \quad 2.722 \mathrm{e}-01 \quad 0.6570 .511644$ tam_loc05 $\quad 1.558 \mathrm{e}-01 \quad 1.527 \mathrm{e}-01 \quad 1.0200 .308622$ tam_loc06 $\quad 9.760 \mathrm{e}-02 \quad 2.584 \mathrm{e}-01 \quad 0.3780 .705984$ tam_loc07 2.452e-01 2.132e-01 1.1500 .251075 tam_loc08 $\quad 2.111 \mathrm{e}-01 \quad 1.888 \mathrm{e}-01 \quad 1.1180 .264580$ tam_loc09 $\quad 1.719 \mathrm{e}-01 \quad 1.847 \mathrm{e}-01 \quad 0.9310 .352786$ tam_loc10 $\quad 5.779 \mathrm{e}-02 \quad 1.855 \mathrm{e}-01 \quad 0.3120 .755570$ tam_loc11 $1.842 \mathrm{e}-01 \quad 1.695 \mathrm{e}-01 \quad 1.0860 .278293$ tam_loc12 $1.648 \mathrm{e}-01 \quad 1.750 \mathrm{e}-01 \quad 0.9420 .347187$ tam_loc13 $\quad 1.368 \mathrm{e}-01 \quad 1.726 \mathrm{e}-01 \quad 0.793 \quad 0.428484$ tam_loc14 $\quad 1.280 \mathrm{e}-01 \quad 1.727 \mathrm{e}-01 \quad 0.7410 .459211$ tam_loc15 $\quad 1.069 \mathrm{e}-01 \quad 1.728 \mathrm{e}-01 \quad 0.6190 .536610$ dis_elec1 $-2.590 \mathrm{e}+00$ 7.140e-01 $-3.6280 .000343 * * *$ dis_elec2 $1.263 \mathrm{e}-01 \quad 1.019 \mathrm{e}+00 \quad 0.1240 .901443$ dis_elec9 $2.466 \mathrm{e}+00 \quad 1.392 \mathrm{e}+00 \quad 1.7720 .077497$. dis_tele1 $2.879 \mathrm{e}+00 \quad 5.542 \mathrm{e}-01 \quad 5.1954 .09 \mathrm{e}-07^{* * *}$ dis_refr1 -7.663e-01 2.719e-01 $-2.8180 .005193 * *$ dis_lava1 $1.348 \mathrm{e}-01 \quad 2.150 \mathrm{e}-01 \quad 0.6270 .531184$ dis_comp1 2.191e-01 2.949e-01 0.7430 .458165 ticlahog11 -6.595e-01 8.092e-01 -0.815 0.415759 ticlahog12 $-2.637 e+00 \quad 1.089 e+00-2.4220 .016112 *$ ticlahog13 $1.417 \mathrm{e}+015.489 \mathrm{e}+00 \quad 2.5820 .010371 *$ $--$

Signif. codes: $0^{‘ * * \prime} 0.001^{* * *} 0.01^{\prime * \prime} 0.05^{\prime \prime} 0.1^{\prime \prime} 1$

Residual standard error: 13460 on 264 degrees of freedom Multiple R-squared: 0.5706, Adjusted R-squared: 0.5137 
F-statistic: 10.02 on 35 and $264 \mathrm{DF}, \mathrm{p}$-value: $<2.2 \mathrm{e}-16$

Tabla 6. sintaxis del modelo de regresión PRI

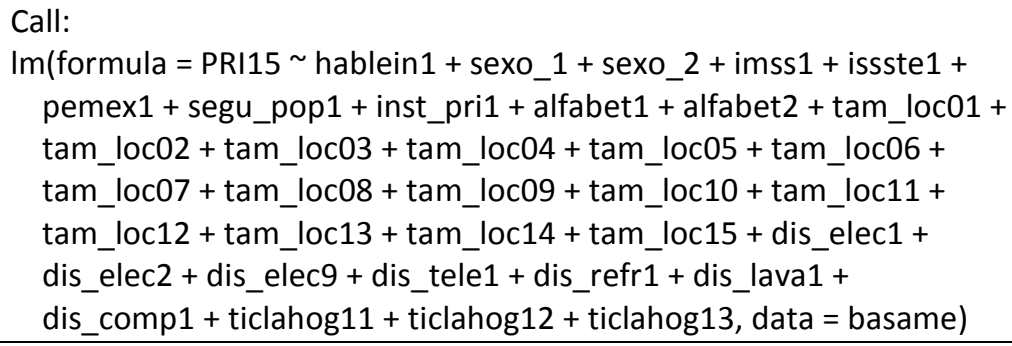

Elaboración propia

Residuals:

Min 1Q Median 3Q Max

$\begin{array}{lllll}-34239 & -7819 & -516 & 8158 & 39127\end{array}$

Coefficients:

Estimate Std. Error t value $\operatorname{Pr}(>|\mathrm{t}|)$

(Intercept) $-1.098 \mathrm{e}+03 \quad 8.897 \mathrm{e}+03-0.1230 .90188$

hablein1 5.465e-02 4.961e-02 $1.102 \quad 0.27168$

sexo_1 7.087e-02 $4.051 \mathrm{e}-01 \quad 0.1750 .86126$

sexo_2 $\quad-3.444 \mathrm{e}-01 \quad 3.896 \mathrm{e}-01 \quad-0.8840 .37746$

imss1 $1.099 \mathrm{e}-01 \quad 4.430 \mathrm{e}-02 \quad 2.481 \quad 0.01374$ *

issste1 $\quad 4.021 \mathrm{e}-02 \quad 1.080 \mathrm{e}-01 \quad 0.372 \quad 0.70997$

pemex1 $\quad-1.255 \mathrm{e}-01 \quad 1.424 \mathrm{e}-01 \quad-0.8810 .37903$

segu_pop1 2.232e-02 3.894e-02 0.5730 .56706

inst_pri1 $1.428 \mathrm{e}-01 \quad 1.805 \mathrm{e}-010.7910 .42955$

alfabet1 $\quad 5.245 \mathrm{e}-01 \quad 4.001 \mathrm{e}-01 \quad 1.3110 .19099$

alfabet2 $\quad 3.853 e-01 \quad 4.093 e-01 \quad 0.941 \quad 0.34738$

tam_loc01 -2.051e-01 4.200e-01 -0.4880 .62575$

tam_loc02 $-5.759 \mathrm{e}-01 \quad 7.144 \mathrm{e}-01 \quad-0.806 \quad 0.42090$

tam_loc03 $7.578 \mathrm{e}-02 \quad 1.957 \mathrm{e}-01 \quad 0.387 \quad 0.69894$

tam_loc04 $-2.140 \mathrm{e}-01 \quad 2.732 \mathrm{e}-01 \quad-0.783 \quad 0.43414$

tam_loc05 2.641e-02 $1.532 \mathrm{e}-01 \quad 0.172 \quad 0.86332$

tam_loc06 -4.762e-02 2.593e-01 -0.1840 .85444$

tam_loc07 $5.498 \mathrm{e}-02 \quad 2.139 \mathrm{e}-01 \quad 0.257 \quad 0.79739$

tam_loc08 -1.405e-02 $1.895 \mathrm{e}-01 \quad-0.0740 .94095$

tam_loc09 $-1.777 \mathrm{e}-01 \quad 1.853 \mathrm{e}-01 \quad-0.9590 .33854$

tam_loc10 -9.657e-02 $1.861 \mathrm{e}-01 \quad-0.5190 .60428$

tam_loc11 $-6.521 \mathrm{e}-02 \quad 1.701 \mathrm{e}-01 \quad-0.3830 .70179$

tam_loc12 $-9.201 \mathrm{e}-02 \quad 1.756 \mathrm{e}-01 \quad-0.5240 .60076$

tam_loc13 $-9.849 \mathrm{e}-02 \quad 1.732 \mathrm{e}-01 \quad-0.5690 .57000$

tam_loc14 -1.050e-01 $1.733 \mathrm{e}-01-0.6060 .54514$

tam_loc15 -1.231e-01 $1.734 \mathrm{e}-01 \quad-0.7100 .47861$

dis_elec1 $2.540 \mathrm{e}-01 \quad 7.166 \mathrm{e}-01 \quad 0.354 \quad 0.72329$

dis_elec2 $2.376 \mathrm{e}+00 \quad 1.023 \mathrm{e}+00 \quad 2.3230 .02092$ *

dis_elec9 $2.087 \mathrm{e}+001.397 \mathrm{e}+001.4940 .13625$

dis_tele1 $9.075 \mathrm{e}-01 \quad 5.562 \mathrm{e}-01 \quad 1.6320 .10393$

dis_refr1 $-1.056 \mathrm{e}-01 \quad 2.729 \mathrm{e}-01 \quad-0.387 \quad 0.69921$

dis_lava1 $8.124 \mathrm{e}-02 \quad 2.157 \mathrm{e}-01 \quad 0.377 \quad 0.70681$

dis_comp1 -8.732e-01 2.960e-01 -2.9500 .00346 **

ticlahog11 $-1.463 \mathrm{e}+00 \quad 8.121 \mathrm{e}-01-1.8020 .07273$

ticlahog12 $-2.279 \mathrm{e}+00 \quad 1.093 \mathrm{e}+00 \quad-2.0860 .03795$ *

ticlahog13 $-1.434 \mathrm{e}+015.508 \mathrm{e}+00-2.6030 .00977 * *$ 
Signif. codes: $00^{* * * \prime} 0.001^{* * *} 0.01^{\prime * \prime} 0.05{ }^{\prime \prime} 0.1^{\prime \prime} 1$

Residual standard error: 13510 on 264 degrees of freedom

Multiple R-squared: 0.5035, Adjusted R-squared: 0.4376

F-statistic: 7.648 on 35 and 264 DF, p-value: $<2.2 e-16$

Tabla 7. Sintaxis del modelo de regresión PRD

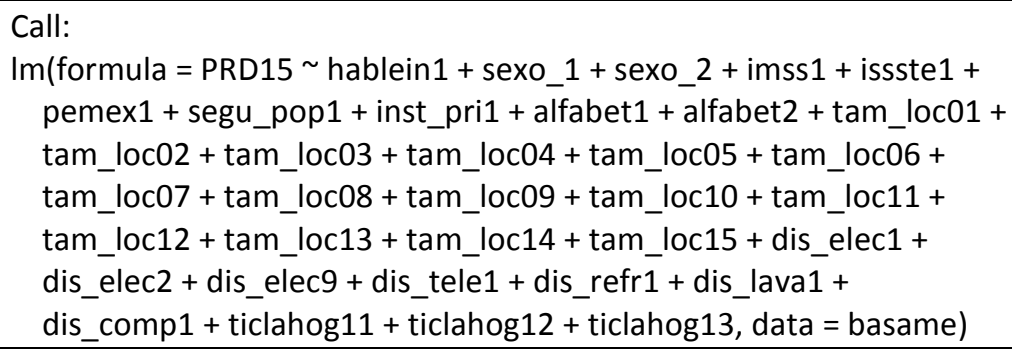

Elaboración propia

Residuals:

Min 1Q Median 3Q Max

$\begin{array}{lllll}-22468 & -7018 & -2197 & 4447 & 41057\end{array}$

Coefficients:

Estimate Std. Error $\mathrm{t}$ value $\operatorname{Pr}(>|\mathrm{t}|)$

(Intercept) 8304.68105 7250.78113 1.1450 .253105

hablein1 $\quad-0.04562 \quad 0.04050-1.1260 .260990$

sexo_1 $0.86674 \quad 0.329702 .6290 .009071 * *$

sexo_2 $\quad 0.89158 \quad 0.321892 .7700 .006008 * *$

imss1 $\quad-0.18731 \quad 0.03613-5.1844 .33 \mathrm{e}-07 * * *$

issste1 $\quad \begin{array}{llll}0.18116 & 0.08819 & 2.054 & 0.040951 *\end{array}$

pemex1 $\quad-0.04871 \quad 0.11596-0.4200 .674800$

segu_pop1 $0.01954 \quad 0.032150 .6080 .543906$

inst_pri1 $\quad 0.07505 \quad 0.14687 \quad 0.5110 .609779$

$\begin{array}{lllll}\text { alfabet1 } & -0.36444 & 0.32784 & -1.112 & 0.267311\end{array}$

alfabet2 $\quad-0.28583 \quad 0.33699 \quad-0.8480 .397106$

tam_loc01 -1.43186 $0.34713-4.1254 .98 \mathrm{e}-05 * * *$

tam_loc02 $-0.44970 \quad 0.58243-0.7720 .440745$

tam_loc03 $-0.65752 \quad 0.15931-4.1274 .93 e-05 * * *$

tam_loc04 $-0.62631 \quad 0.22337-2.8040 .005425 * *$

tam_loc05 $-0.59635 \quad 0.12470-4.782$ 2.89e-06***

tam_loc06 $-0.60208 \quad 0.21105-2.8530 .004678 * *$

tam_loc07 $-0.64950 \quad 0.17452-3.7220 .000242 * * *$

tam_loc08 $-0.575520 .15420-3.7320 .000233 * * *$

tam_loc09 $-0.65401 \quad 0.15089-4.3342 .08 \mathrm{e}-05 * * *$

tam_loc10 $-0.624650 .15176-4.1165 .16 \mathrm{e}-05 * * *$

tam_loc11 $-0.60765 \quad 0.13867-4.3821 .70 \mathrm{e}-05 * * *$

tam_loc12 $-0.60034 \quad 0.14298-4.1993 .67$ e-05 ***

tam_loc13 $-0.60675 \quad 0.14104-4.302$ 2.39e-05***

tam_loc14 $-0.606090 .14113-4.295$ 2.46e-05***

tam_loc15 $-0.59883 \quad 0.14127-4.2393 .11 \mathrm{e}-05 * * *$

dis_elec1 $\quad-0.562420 .58312-0.9650 .335681$

dis_elec2 $\quad 1.475790 .83595 \quad 1.7650 .078657$.

dis_elec9 $\quad-0.47742 \quad 1.14370-0.4170 .676702$

dis_tele1 $\quad-0.35421 \quad 0.45266 \quad-0.7830 .434619$ 


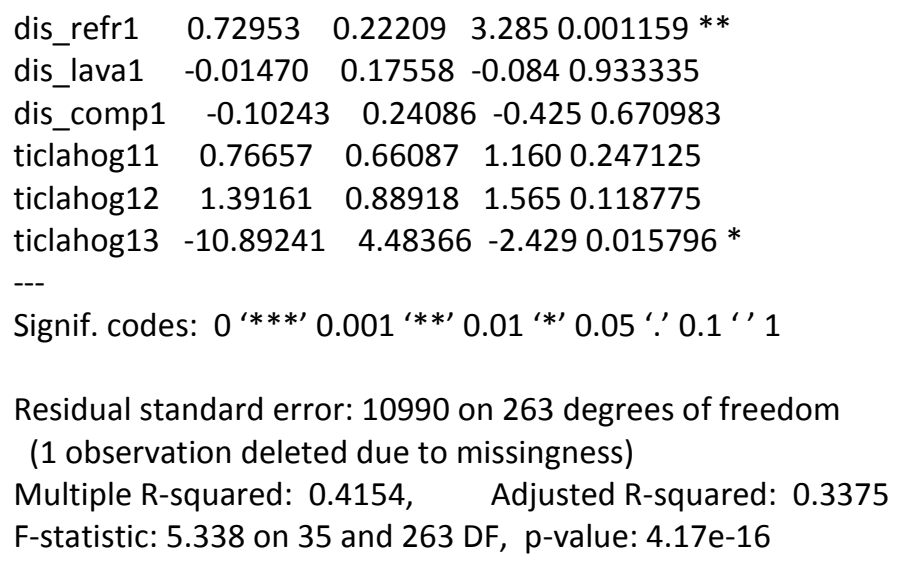

\section{Discusión}

El desarrollo de la geografía electoral en México ha sido una aportación de varios investigadores, principalmente sociólogos, politólogos y geógrafos, entre los que se encuentra Martínez Assad (1990) y Molinar Horcasitas (1990) quienes en el año de 1990 analizan con una gran agudeza los cambios territoriales que han afectado los resultados electorales en la crisis del fordismo, cuando el Estado va perdiendo el monopolio corporativo de los diferentes grupos sociales y se va encumbrando el posfordismo, que determina al Estado a cumplir con una serie de reformas políticas flexibles que garantizan la pluralidad política y enaltecer las libertades individuales y de los mercados, pero al poco tiempo de andar concentran la renta, retienen los salarios, conceden poder incontrolable a los mercados financieros y reparten miseria y riqueza extravagante.

Liliana López Levi (2006) señala que el problema es regionalizar sin perder de vista un cúmulo de componentes, votantes de diferentes ideologías, clase social e identidad cultural; simultáneamente, considerar la imparcialidad en las fronteras electorales. Dilema en el que convergen metodologías, técnicas y políticas. Posteriormente, Gómez Tagle y Valdés (2000) resaltan características de la población, su dispersión y concentración en las preferencias electorales.

Trelles, et. al $(2016 ; 2015)$ señalan que para cerrar el ejercicio técnico de la distritación en México, se requiere construir herramientas de análisis que permitan al usuario acceder, manipular, operar y comprender la información demográfica electoral. Señalan que el usuario no tiene acceso a la cartografía preliminar, ni tampoco a las contrapropuestas de los partidos 
políticos, involucrados en el proceso de redistritación, lo que también impulsaría la transparencia en la información y no sólo el análisis geoestadístico con los datos finales.

La relación numérica entre el total de diputaciones y el total de población en un distrito debe lograrse con equilibrio para que cada individuo tenga la confianza de elegir a un diputado (López, 2006). Lo justo es que haya un equilibrio demográfico entre los distritos.

López (2006) señaló que en el año 2004 el IFE mostró que 34\% de los distritos estaban por encima del rango de tolerancia establecido en 1996, situación que impulsó una redistritación concluida en el año 2005.

Cabe señalar que el territorio es dinámico por lo cual se debe actualizar periódicamente las distritaciones. Cuidando que el ejercicio no favorezca o reduzca las preferencias electorales.

Los criterios fundamentalmente geográficos que se tomaron en cuenta en la redistritación de 2005 fue la contigüidad, procurando integridad con las unidades territoriales vecinas sin romper los vínculos de identidad. El problema técnico radica en la relación entre la población por distrito y la relación entre la media poblacional estatal.

El Instituto Nacional Electoral no ha conseguido sistematizar la manera en que se incorpora el conocimiento local indispensable para conocer la integridad de las comunidades y el de tiempos de traslado. No existe correspondencia entre los distritos electorales federales y locales, lo que propiciaría la integración territorial, la identidad ciudadana y facilitaría tareas operativas. Permitiendo el conocimiento territorial por parte de los grupos locales (LÓPEZ y REYES, 2008; LACOSTE, 1977).

Indudable es que se ha realizado un excelente análisis de los distritos por parte de la comunidad científica, que ha permitido ir mejorando la calidad técnica de los mismos, sin embargo, cuando se avanza en la pluralidad democrática en ciertas regiones se agrupan los distritos y por el contrario donde se va perdiendo el armazón monopólico de las corporaciones partidistas se amplia el número de distritos.

De esta manera la fineza técnica en los distritos no desplaza la necesidad política de los grupos sociales por incorporarse en esta acción que reconfigura los límites normativos y de la vida en común. 


\section{Conclusiones}

La práctica de ordenamiento territorial electoral se mostró como un ejercicio favorable para corregir las fallas en la democracia mexicana. Actividad que debe ser permanente, puesto que la sociedad en el tiempo va cambiando su comportamiento al que se deben orientar esfuerzos para alcanzar una justa distribución y extirpar las patologías sociales que desencadenan crisis económica, social y de identidad. El secreto de mantener el poder por parte de los grupos hegemónicos radica en la unilateralidad técnica que cada vez es más fina, pero que no es transparente en sus métodos y excluye de la participación a los movimientos sociales para tomar parte en la observación y vigilancia de la definición de los distritos.

Como se mencionó, antes de la distritación de 1996 se tenían deficiencias en la cartografía, mismas que no daban cuenta de las modificaciones en el espacio. Corregir las grietas representaba un avance en el ejercicio de redistribución del poder en el Legislativo.

No se han realizado esfuerzos por coordinar los distritos federales con los distritos locales, produciendo una barrera para la democracia en la cotidianidad. El proceso, más que un esfuerzo absolutamente técnico, objetivo y positivo, para garantizar el escenario de redistribución, se orientó por el lado contrario, concentró el poder y refrendó, a través de la redistritación del año 2005 y 2017, la fórmula: "el poder se comparte, no se reparte". Así mismo, el poder, tiene como centro el secreto público que consiste en realizar fraudes a través de procedimientos técnicos que dificultan el entendimiento y se colocan en el ámbito de la falta de transparencia.

Son la Ciudad de México, el Estado de México y Veracruz, los tres estados que con mayor fuerza ampliaron o recortaron sus polígonos para generar o disminuir más distritos. Dichos estados, desde 1996, tienen una reconfiguración en la representación partidaria, aunque eso no signifique mayor poder para los electores. En la redistritación de 2005 y 2017 se hicieron los ajustes, por ejemplo, en el Estado de México, para incrementar el número de distritos, lo cual significa más poder en el Legislativo, resarciendo la afectación que provoca el triunfo de otros partidos políticos, diferentes a la hegemonía del PRI que durante el siglo XX conservó y concentró el poder.

Los electores desconocen la importancia que tiene la organización del espacio para las elecciones. De forma ingenua, se muestra a la población que emitir y omitir un voto tiene el 
mismo peso al realizarlo en cualquier parte de la República y perteneciendo a cualquier clase social. Asimismo, la población desconoce o confunde las escalas administrativas: colonia, municipio, entidad y República, con las escalas electorales como: casilla, secciones y distritos. El Instituto Nacional Electoral precisa mostrar cada uno de los procesos de distritación y hacer partícipe a los grupos sociales, de otra manera será una estructura burocrática que administra la simulación para mantener el poder.

Las condiciones de distritación tienen que ver más con factores económicos, como los intercambios, flujos y la fijación de soportes materiales para espacios productivos que sólo las variables del Censo de Población y Vivienda, los cálculos de movilidad y accesibilidad. Los distritos más amplios se localizan en los lugares donde se está ofertando gran cantidad de terreno a las compañías inmobiliarias, los distritos con mayor población se encuentran en las zonas fronterizas del país, donde se están insertando el mayor número de maquiladoras y vías de comunicación para el posicionamiento global. No hay relación entre los distritos más amplios y la mayor cantidad de población. En algunos estados de la República como por ejemplo el Estado de México se retrata el fenómeno conocido como salamandra.

Los factores de la vivienda y poblacionales que impactan de una forma más significativa en la variación de la votación por los tres principales partidos son: la seguridad social a la familia del trabajador (Imss), analfabetismo y disposición de televisión. Se infirió que la base trabajadora en su esfuerzo por permanecer integrada en el modelo de desarrollo, participa en la elección de los cuadros que modelan el territorio para permanecer en el poder.

El software R para estadística es una herramienta útil, innovadora y práctica para observar, explicar y analizar fenómenos sociales como la participación electoral, diseño cartográfico para la participación y características de los participantes.

Las tablas, figuras y regresiones permitieron observar demográficamente grupos etarios con preferencia de partido, además, inferir y proyectar fenómenos ligados como la emigración, la localización de los distritos y fueron prueba contundente para aceptar la hipótesis alterna. El programa R, igualmente, arrojó los coeficientes y puso a disposición, del análisis visual, elementos gráficos. 


\section{Bibliografía}

ADLER, J. 2010. $R$ in A Nutshell. A Desktop Quick Reference. California: O'Reilly.

AHO, K. 2014. Foundational an Applied Statistics for Biologists using R. Electronic Appendix (Introduction to R). New York: Taylor \& Francis Group.

ARNOLD, T. and TILTON, L. 2015. Humanities Data in R. Exploring Networks, Geospatial Data Images, and Tex. New York: Springer.

BEAUJEAN, A. 2014. Latent Variable Modeling Using R. A Step-by-Step Guide. New York: Routledge.

BILDER, C. and LOUGHIN, T. 2015. Analysis of Categorical Data with R. New York: Taylor \& Francis Group.

BIVAND, R.; PEBESMA, E.; GÓMEZ-RUBIO, V. 2013. Applied Spatial Data Analysis with R. New York: Springer.

CHANG, W. 2013. $R$ Graphics Cookbook. California: O'Reilly.

COLL-HURTADO, A. 2008. "Geografía, territorio y sociedad". En: 1er Coloquio Internacional Geografía Electoral. 14 y 15 de mayo del 2008. México: Instituto Federal Electoral, Instituto de Geografía, Universidad Nacional Autónoma de México.

COTTON, R. 2013. Learning R. California: O'Reilly.

DE OLIVEIRA, F. 1982. Elegía para una re(li)gión, Sudene, Nordeste. Planificación y Conflictos de Clase. México, Fondo de Cultura Económica. p. 9-48.

FROMM, E.; MACCOBY, M. 2007. Sociopsicoanálisis del campesino mexicano. México: Fondo de Cultura Económica.

GAETAN, C.; GUYON, X. 2010. Spatial Statistics and Modeling. New York: Springer.

GÓMEZ TAGLE, S.; VALDÉS, M. E. 2000. La geografía del poder y las elecciones en México. México: Instituto Federal Electoral-Plaza y Valdés.

IFE. 2005. Distritación 2004-2005: camino para la democracia. México: Instituto Federal Electoral.

IFE. 2015. Tipología de los distritos electorales. México: Instituto Federal Electoral.

IFE. 2005a. Cartografía digital a escala distrital 1996. México: Instituto Federal Electoral.

IFE. 2005b. Cartografía digital a escala distrital 2005. México: Instituto Federal Electoral.

INE. 2017. Concentrado General Nacional de los Distritos. México: Instituto Nacional Electoral.

INEGI. 2010. Censo de Población y Vivienda 2010. México: Instituto Nacional de Estadística Geografía e Informática.

LACOSTE, Y. 1977. La geografía: un arma para la guerra. Barcelona: Editorial Anagrama.

LÓPEZ, L. 2006. Redistritación electoral en México: logros pasados y retos futuros. Investigaciones Geográficas, Boletín del Instituto de Geografía, Núm. 61, p. 91-113.

LÓPEZ, L.; REYES, E. 2008. Federalismo y redistritación electoral en México. Política y Cultura, primavera, Núm. 29, p. 125-147. 
LUNA, L. 2016a. Escalas y determinaciones en la reforma a la propiedad de los energéticos: México. Revista electrónica de estudios latinoamericanos. 14, 54, pp. 38-55.

LUNA, L. 2016b. Representação da refinaría Bicentenário: Hidalgo, México. Estudos Geográficos, Rio Claro, 14(1): 62-78, jan./jun.

LUNA, L. 2017. Representação e produção do espaço: duas conceituações analisadas por Henri Lefebvre. Diaphonía, v. 3, n. 1.

LUNA, L. 2014. La desvalorización de la tierra en el patrón de producción, agudizada por las reformas energética y laboral de Enrique Peña Nieto. Estudios Socioterriotoriales. Revista de Geografía. Núm. 16, vol. 1, jul-dic 2014, p. 47-80.

MARTÍNEZ ASSAD, C. 1990. Balance y perspectivas de los estudios regionales en México. México: UNAM-CEIICH-Porrúa.

MOLINAR HORCASITAS, J. 1990. "Geografía electoral”, en Carlos Martínez Assad, Balance y perspectivas de los estudios regionales en México. México: UNAM-CEIICH-Porrúa.

MEIXUEIRO, G.; y BACA, A. 2013. La reforma energética en la opinión pública. México: Centro de Estudios Sociales y de Opinión Pública. p. 29.

PATHAK, M. 2014. Beginning Data Science with R. New York: Springer.

SANTOS, M. 2000. La naturaleza del espacio: técnica y tiempo. Razón y emoción. Barcelona: Ariel.

TAUSSIG, M. 2015. La magia del Estado. México: Siglo XXI.

TEETOR, P. 2011. $R$ Cookbook. California: O’Reilly.

TRELLES, Alejandro, ALTMAN, M.; MAGAR, E.; MCDONALD, M. 2015. "Transparency, Automated Redistricting, and Partisan Strategic Interaction: The Case of Mexico", ponencia presentada en el Electoral Integrity Workshop, San Francisco, septiembre.

TRELLES,A.; ALTMAN, M.; MAGAR, E; McDONALD, M. 2016. “Datos abiertos, transparencia y redistritación en México". Política y gobierno. Vol. XXIII, n. 2, p. 331-364.

Tramitação do artigo na revista

Submetido: 02/04/2018

Revisões requeridas: 05/05/2018

Versão revista: 20/05/2018

Aceito: 25/05/2018 